\title{
Stimulus intensity and the visual generalization gradient to wavelength in the pigeon'
}

JOZEF COHEN

UNIVERSITY OF IL LINOIS

Visual generalization gradients to wavelength in the pigeon have been measured with stimuli differing in both wavelength and intensity. The gradient attributed to changes in wavelength may be due to differences in intensity. The original Guttman-Kalish experiment was duplicated essentially, but using test stimuli at two intensities at each wavelength. The gradients for the two intensities are nearly identical. Visual generalization gradients may be attributed safely to wavelength.

There is a large experimental literature based on the investigation by Guttman \& Kalish (1956) wherein the visual generalization gradient to wavelengths in pigeons was determined. The Guttman and Kalish study, and all similar studies, suffer from a subtle defect-that the intensity of the test stimuli were uncontrolled; Guttman and Kalish wrote: "No attempt was made to produce an equal energy spectrum or alternatively to equate spectrum lights in terms of the visibility function."

Since the intensity output of the monochromator will ordinarily be quite different for the several test stimuli (it depends on the emission spectrum of the source, characteristics of the grating or prism, etc.), a change in wavelength will be accompanied by a change in intensity. The visual generalization gradient, therefore, may be a function of wavelength, of intensity, or of both, and cannot be assumed to be a function of wavelength alone. Intensity may well be a relevant factor, for an investigation by Blough (1959) has demonstrated that a gradient for intensity level occurs when wavelength is held constant.

The Investigation described here measures the effects of intensity on the visual generalization gradient by repeating the Guttman-Kalish experiment essentially, but using test stimuli at two different intensities at each wavelength. If the high intensity gradient differs substantially from the low intensity gradient, intensity has an effect. If the high intensity gradient differs little from the low intensity gradient, intensity has no effect.

\section{Method and Procedure}

This experiment used 15 domestic male white Carneaux pigeons, maintained at $65-70 \%$ of their free feeding weights. The birds were without prior experimental histories.

A Bausch and Lomb high intensity grating monochromator, utilizing $10 \mathrm{~m} \mu$ entrance and exit slits, projected near-monochromatic lights onto a trans- luscent key in the bird's chamber. A filter holder, between the monochromator and the key, held a Wratten neutral filter of either $75 \%, 50 \%$, or $25 \%$ transmission.

The animal chamber was of standard size, with a $3 / 4$ in. transluscent key. The chamber was without illumination except that emerging from the key. The food hopper was illuminated, with a mirror, by the same monochromatic light falling onto the key.

Massey-Dickinson modules programmed the schedules and recorded the responses.

Each pigeon was first shaped, under room illumination, to peck at the key for a food reinforcement. A near-monochromatic stimulus of $570 \mathrm{~m} \mu$ was placed on the key, with the Wratten filter of $50 \%$ transmission inserted, serving as the discriminative stimulus. The training period each day consisted of $151-\mathrm{min}$ periods with the stimulus on, with a 20-sec blackout between periods. The initial continuous schedule was gradually changed, over 60 days, to VR 50 which was maintained for nine additional days. On the day following, each bird was given five "warm-up" periods with the discriminative stimulus. Then each bird was tested twice with near-monochromatic stimuli at $\mathbf{5 5 0}$ $\mathrm{m} \mu, 560 \mathrm{~m} \mu, 570 \mathrm{~m} \mu, 580 \mathrm{~m} \mu$, and $590 \mathrm{~m} \mu$ with the Wratten filter of $75 \%$ inserted (the high intensity stimuli), and twice with stimuli at the same wave-

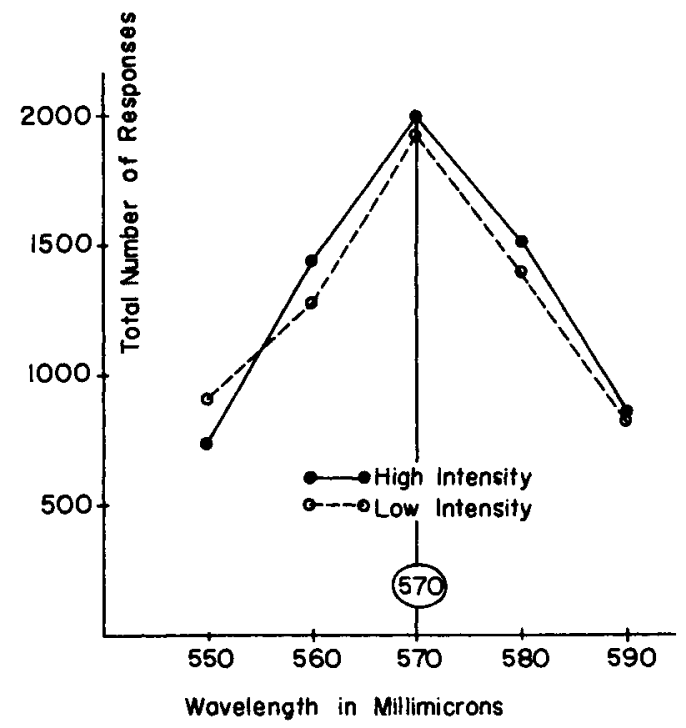

Fig. 1. Total responses of 15 pigeons to five test stimuli of high intensity and to five test stimuli of low intensity, after training to a stimulus of $570 \mathrm{~m} \mu$ at intermediate intensity. 
lengths with the Wratten filter of $25 \%$ inserted (the low intensity stimuli), in random order; there were, then, a total of 20 test periods of 1 min with a 20-sec blackout between.

\section{Results and Discussion}

The combined responses of 15 pigeons to the five near-monochromatic test stimuli of low intensity are shown in Fig. 1. The high intensity gradient and the low intensity gradient are almost identical. It is concluded that pigeons generalize to wavelength changes when wavelength is changed, and disregard accompanying changes of intensity. The GuttmanKalish generalization gradient may be viewed as a function of wavelength alone.

\section{References}

Blough. D. S. Generalization and preference on a stimulus-intensity continuum. J. exp. Anal. Behav., 1959, 2, 307-317.

Guttman, N., Kalish, H. I. Discriminability and stimulus generalization. J. exp. Psychol., 1956, 51, 79-88.

\section{Note}

1. This research was supported by the U. S. Department of Health, Education, and Welfare under grant number MH 08922-01-02. 